\title{
Obstacle Avoidance with Virtual Sensor in Mobile Robot's Motion using the Advanced Potential Field Controller
}

\author{
Marwa T. Yousef \\ Tebbin Institute for Metallurgical \\ Studies, \\ Tebbin, Cairo, Egypt
}

\author{
Elsayed M. Saad \\ Faculty of Engineering, Helwan \\ University \\ Helwan, Cairo, Egypt
}

\author{
Shahira M. Habashy \\ Faculty of Engineering, Helwan \\ University \\ Helwan, Cairo, Egypt
}

\begin{abstract}
This paper introduces an Advanced Artificial Potential Field (AAPF) controller which is used to control the robot's motion in cluttered environments. The proposed approach gives less computation and increases the reaction speed of the robot at obstacle avoidance situations. The increasing of robot's reaction speed doesn't affect on the smoothness of its path due to the use of Genetic algorithms (GA) which select the optimum factors of the forces applied to the robot. A measure of smoothness is used to guide the genetic algorithm to select forces' factors with minimum smoothness. Of course more smoothness means less distance and more speed to reach the goal. The Advanced controller using GA is simulated with different cases on Windows Vista using Matlab Software. These cases include environments with single obstacle up to three obstacles and multi-knee corridor. Results are compared to previous works.
\end{abstract}

\section{General Terms}

Genetic Algorithm, Robot Motion.

\section{Keywords}

Artificial Potential Field, Obstacle Avoidance, Virtual Sensor.

\section{INTRODUCTION}

Mobile robots is improved from path guidance methods to autonomous mobility, resulting on obstacle avoidance of robots have emerged steadily over the years including directional command methods, such as artificial potential fields [1-5] and speed-space commands, such as the curvature foundation system [6-9]. However, most previous results remain a challenging problem as most existing methods have not considered the mobility of robots and obstacles. These algorithms cause a robot to move very slowly for obstacle avoidance. If a robot moves very slowly, most of the established algorithms can be applied to avoid obstacles. As it moves faster and faster, avoidance control is more difficult and the robot tends to collide more frequently with obstacles. The virtual sensor concept that was introduced in [10] is used in our work. This concept is similar to that of the Doppler Effect. When a robot heads to an obstacle, the distance on the robot sensor is longer than the virtual sensor. Likewise, the physical distance on the robot sensor is shorter than the virtual one when it goes away from an obstacle.

The philosophy of the artificial potential field (APF) approach is described in [1].

In this paper, a modification of the artificial potential field equations will be demonstrated. This modification gives high reaction speed of the robot without any abrupt changes in robot path. Efficiency of robot obstacle avoidance will be regarded too. The Genetic Algorithm (GA) is used also to select the optimum factors of the repulsive and the attractive forces in the offline state which can be then applied on the robot in the online state as in [11]. The optimum factors are those who make the robot motion much smoother. Simulation on Matlab is executed for testing the performance of the AAPF controller in the task of robot obstacle avoidance. The simulation is done at four workspaces as at [10],[11]. The simulation results are then used to compare the performance of the proposed system and the established system in [10], [11] to evaluate the proposed system effectiveness. The odometry of the robot is calculated using the angle approach [12].

This paper is organized as follows. Section 2 illustrates the Advanced Artificial Potential Field (AAPF). Section 3 explains the determination of the optimum factors of the potential field controller forces using GA and illustrates the GA fitness function which is used in our work. Section 4 shows the performance of the proposed system by simulation in four different cases. In addition, comparison with the established system in [10], [11] will be reviewed. Conclusion and future work are given in Section 5.

\section{ADVANCED ARTIFICIAL POTENTIAL FIELD (AAPF)}

As the robot approaches the obstacle the repulsive force $\mathrm{F}_{\mathrm{o}}\left(\mathrm{P}_{\mathrm{r}}(\mathrm{t})\right)$ increases. On the other hand, the attractive force to the goal $F_{g}\left(P_{r}(t)\right)$ will be decreased as it approaches its goal. Therefore, the net forces obtained from the artificial potential fields equations is noted in [1], [10].

Where, the repulsive force is inversely proportional to the distance between the robot and the obstacles. And the attractive force is proportional to the distance between the robot and the goal. This logic rule led us to reform the equation of the repulsive part in the APF which becomes much simple to be computed as in equation (1).

$U_{o}\left(P_{r}(t), P_{O}(t)\right)= \begin{cases}K_{r} \ln (\rho) & \text { if } \rho \leq \rho_{o} \\ 0 & \text { if } \rho>\rho_{o}\end{cases}$

Where: $P_{r}(t)$ is the position of the robot which is calculated by using the angle approach as in [12], $P_{O}(t)$ is the position of the obstacle, $K_{r}$ is the repulsive force factor, $\rho$ is the virtual sensor reading of the distance between the robot and the obstacle which is calculated as in [10], and $\rho_{o}$ is the threshold distance between the robot and the obstacle.

So, the repulsive force equation $\mathrm{F}_{\mathrm{o}}\left(\mathrm{P}_{\mathrm{r}}(\mathrm{t})\right)$ which represents the first derivative of equation (1) becomes 


$$
F_{O}\left(P_{r}(t)\right)_{\mathrm{x}}= \begin{cases}K_{r}\left(\frac{1}{\rho}\right)\left(\frac{\partial \rho}{\partial x}\right) & \text { if } \rho \leq \rho_{o} \\ 0 & \text { if } \rho>\rho_{o}\end{cases}
$$

Where, $F_{O}\left(P_{r}(t)\right)_{x}$ is the repulsive force at $x$-axis. Of course the less computation gives high speed reaction to the robot. While attractive force computation remains as in [10].

\section{DETERMINING THE OPTIMUM \\ FACTORS AND FITNESS FUNCTION}

The attractive and repulsive coefficients $\mathrm{Ka}, \mathrm{Kr}$ of the forces are determined empirically in [10], that shouldn't grantee a fast and smooth robot path. To overcome that empirical factors determination, one of the optimization techniques such as (GA) can be used to better determine those factors.

The fitness function which is used in GA optimizer is the smoothness (SM) that is defined as a criterion of the evaluation to measure the various robot trajectories as:

$$
\begin{gathered}
S M=\sum_{n=0}^{m}\left(\varphi_{n}-\varphi_{n-1}\right) \\
\varphi_{n}=\tan ^{-1} \frac{y_{n}-y_{n-1}}{x_{n}-x_{n-1}}
\end{gathered}
$$

Where $: m=$ the last pose number of robot trajectory, $x_{n}$ and $y_{n}$ represent the robot position at $\mathrm{n}^{\text {th }}$ sampling time. $\varphi_{n}$ is the angle between current and former position at $n^{\text {th }}$ sampling time.

First the optimum parameters for the potential field controller are obtained in offline state. It includes guided random search operation using GA to determine the optimum factors for $\mathrm{Kr}$ and $\mathrm{Ka}$ which are the factors of the repulsive and the attractive forces respectively. Four different environments for testing the improved potential field controller are used. The first environment has one obstacle, the second environment has two obstacles, the third one has three obstacles, finally the fourth has multi-knee corridor. The start point of the robot at the first three workspaces are $(0,0)$, while at the fourth, the start point of the robot is $(2,0)$. The end points (goals) at the four workspaces are $(4,2),(4,3),(4,3)$ and $(6.5,6.5)$ respectively. These cases are those typically used in [10]. Ka and $\mathrm{Kr}$ values for each workspace according to using GA are denoted in Table 1.

Table 1. The optimum values of forces factors at the four cases

\begin{tabular}{|c|c|c|c|c|}
\hline & Case 1 & Case 2 & Case 3 & Case 4 \\
\hline $\mathrm{K}_{\mathrm{a}}$ & 15.203 & 23.5582 & 16.5519 & 16.7876 \\
\hline $\mathrm{K}_{\mathrm{r}}$ & 1.1741 & 0.2756 & 0.1094 & 0.0502 \\
\hline
\end{tabular}

\section{SIMULATION RESULTS}

This section shows the simulation results of robot motion in four different environments at two states. The first state is the robot motion to go to a static goal at each environment using its $\mathrm{Ka}$ and $\mathrm{Kr}$ values. The second state is the robot motion to go to a static goal at each environment using the average values of $\mathrm{Ka}$ and $\mathrm{Kr}$ of the four environments. To show the optimization achieved in the performance of the proposed method, it is compared with the results in [10-11]. Sections 4.1 and 4.2 show the simulation results at these two states respectively.

\subsection{Robot Motion to reach a Static Goal using AAPF controller with optimum Ka and $\mathrm{Kr}$}

The motion paths at the four cases are shown in Fig. 1, 2, 3, 4, respectively. Where: Fig. 1.a, Fig. 2.a, Fig. 3.a and Fig. 4.a represent the simulation results in [10] using APF without GA. Fig. 1.b, Fig. 2.b, Fig. 3.b and Fig. 4.b represent the simulation results in [11] using APF with GA. Fig. 1.c, Fig. 2.c, Fig. 3.c and Fig. 4.c represent the simulation results of the proposed system using AAPF with GA. The motion paths (a) at each figure of figures 1,2, 3, 4 for the four cases present that the robot with empirical values of $\mathrm{Ka}$ and $\mathrm{Kr}$ displays slower evasive action than (b), (c). The robot in (a) of each figure makes abrupt changes in its paths while the robot at (b), (c) display smoother changes. The smoothness achieved with the proposed system is better than that achieved with the previous work. The smoothness achieved at each case is compared with the previous works and is shown in Table 2 . As shown from the Table 2 a large optimization of the smoothness is occurred with the proposed approach. When the path of the robot is smoother it means that the robot takes a short path and less time to go to the goal. And the reaction speed of the robot will be higher at the proposed system than [10], [11].

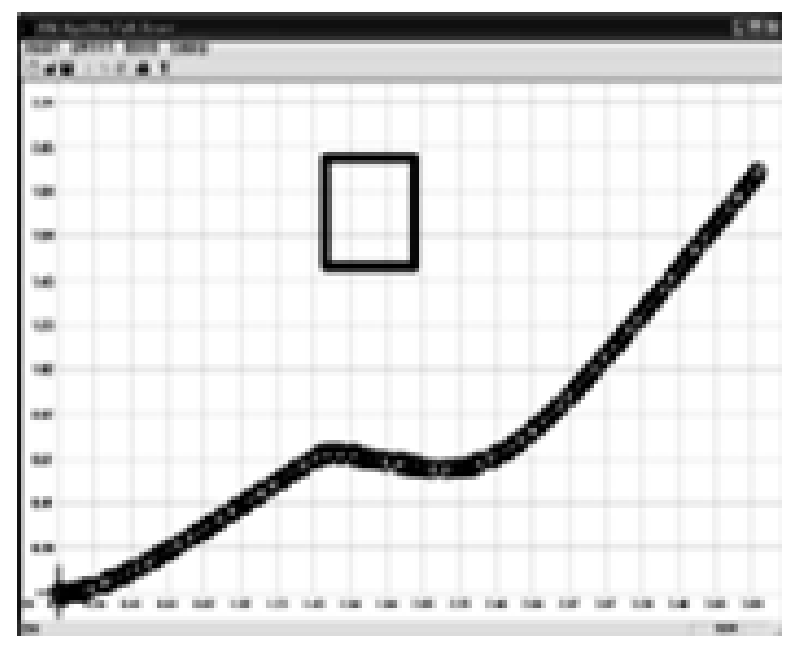

(a)

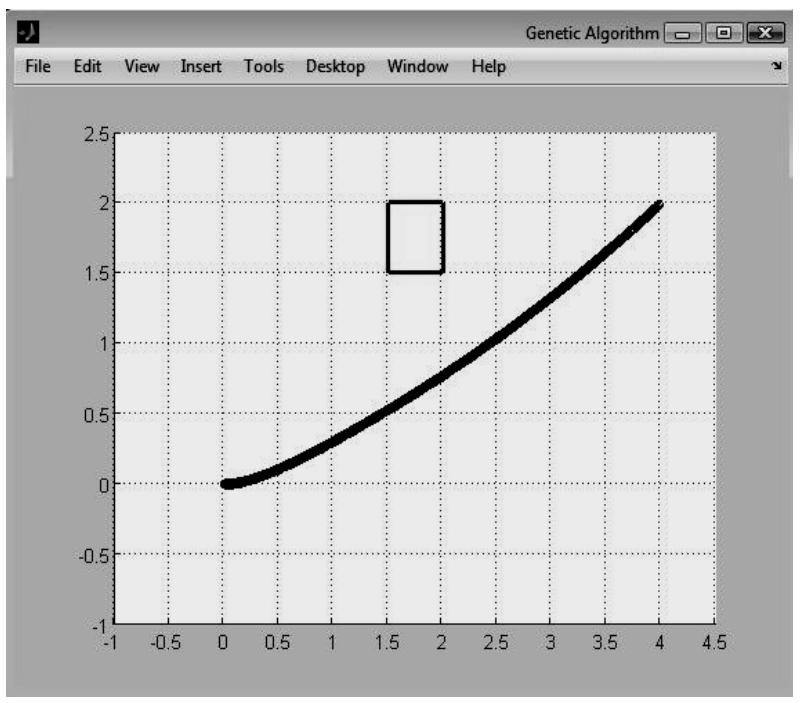

(b) 


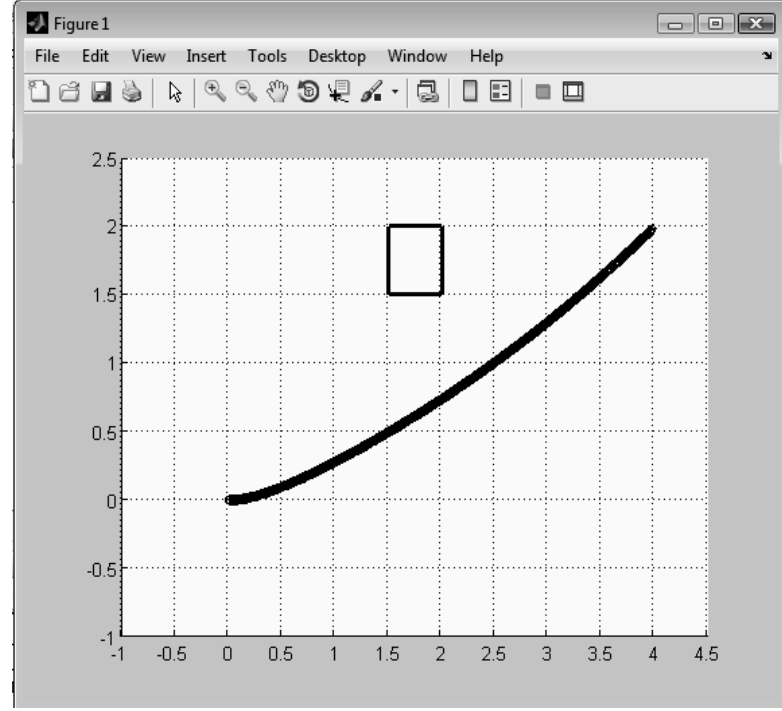

(c)

Fig 1: Robot paths in Case 1 with different systems. (a) using APF without GA in [10], (b) using APF with GA in [11], and (c) the proposed system using AAPF with GA.

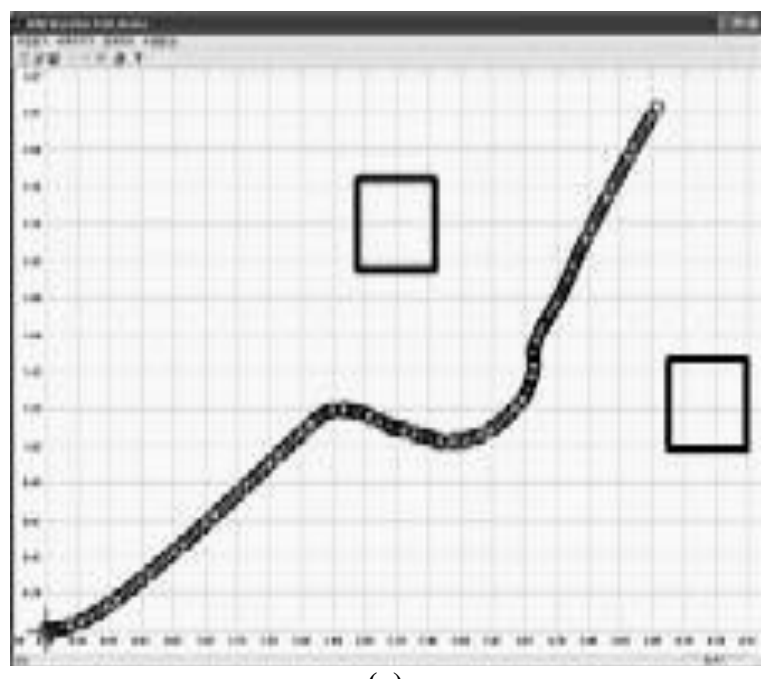

(a)

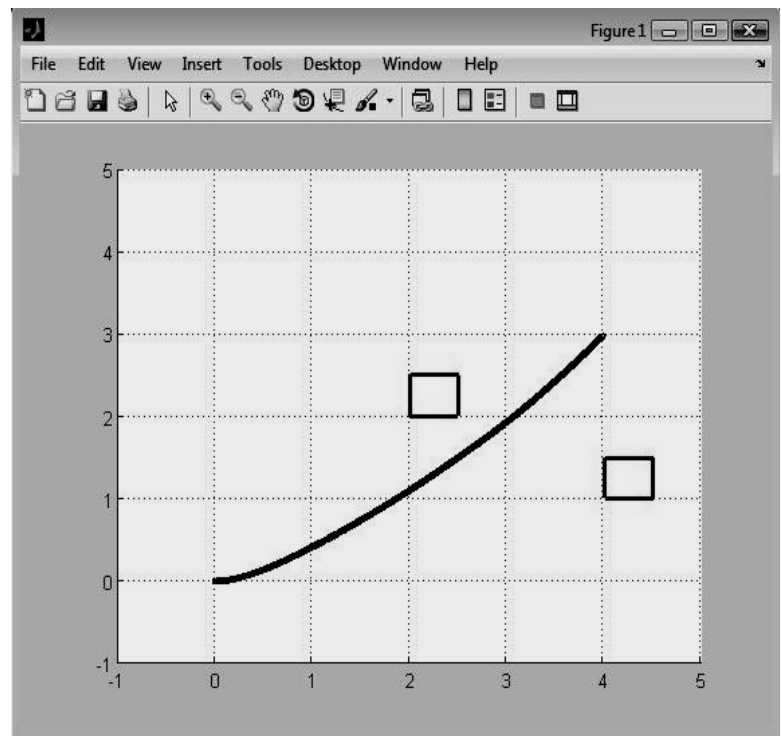

(b)

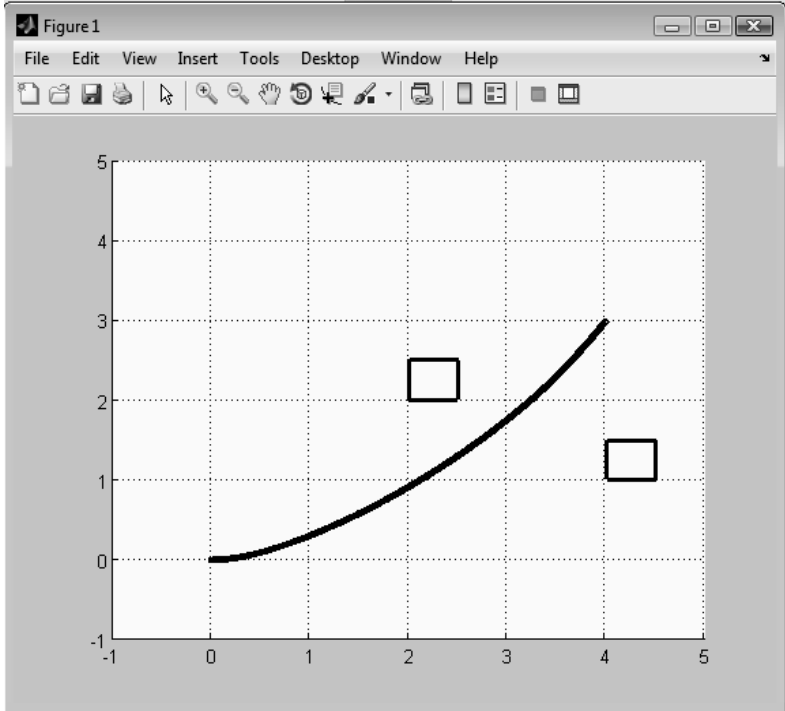

(c)

Fig 2: Robot paths in Case 2 with different systems. (a) using APF without GA in [10], (b) using APF with GA in [11], and (c) the proposed system using AAPF with GA.

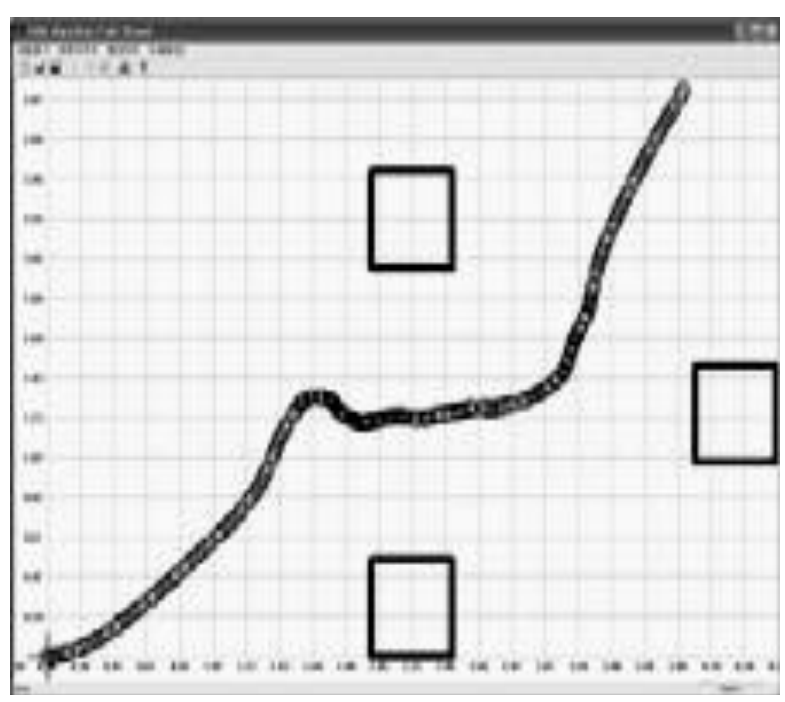

(a)

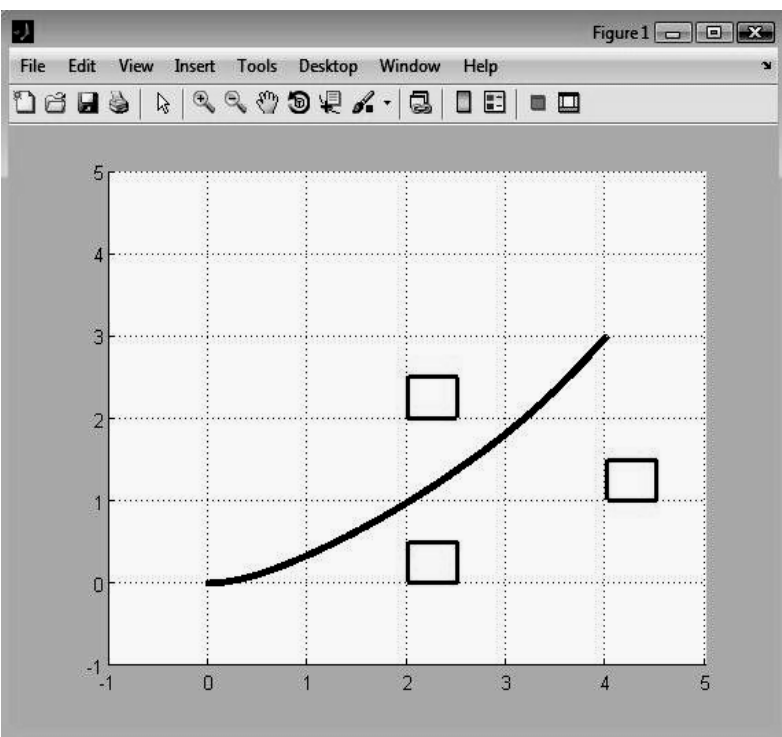

(b) 


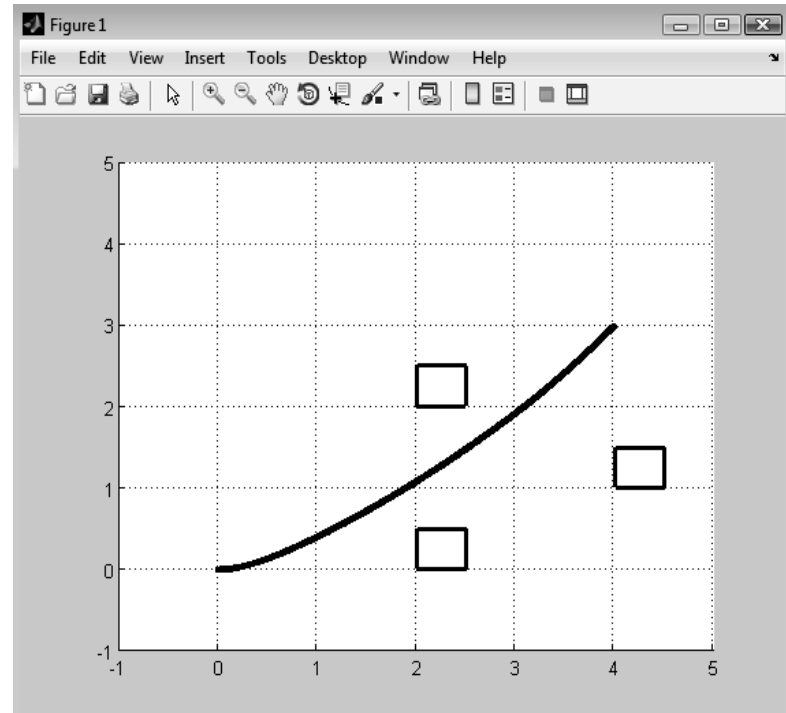

(c)

Fig 3: Robot paths in Case 3 with different systems. (a) using APF without GA in [10], (b) using APF with GA in [11], and (c) the proposed system using AAPF with GA.

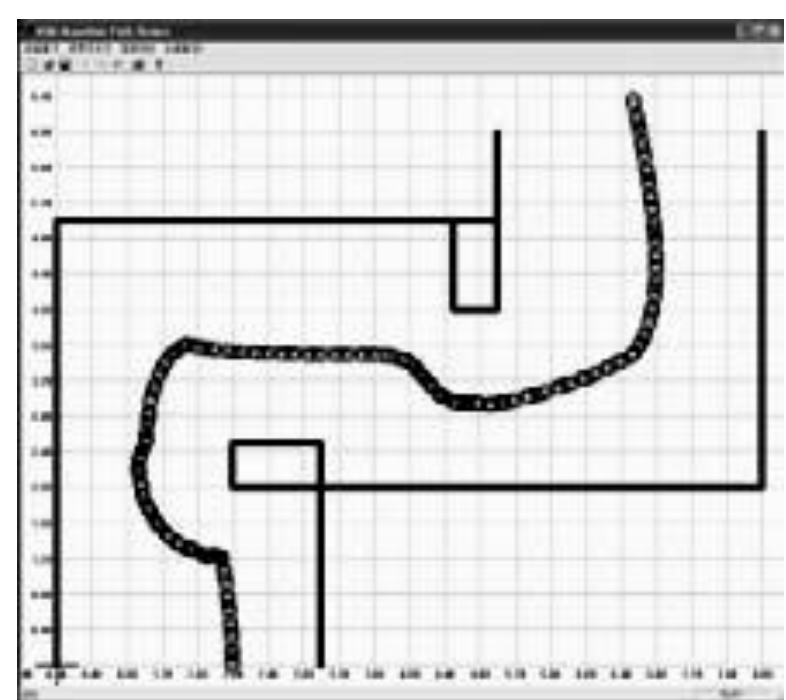

(a)

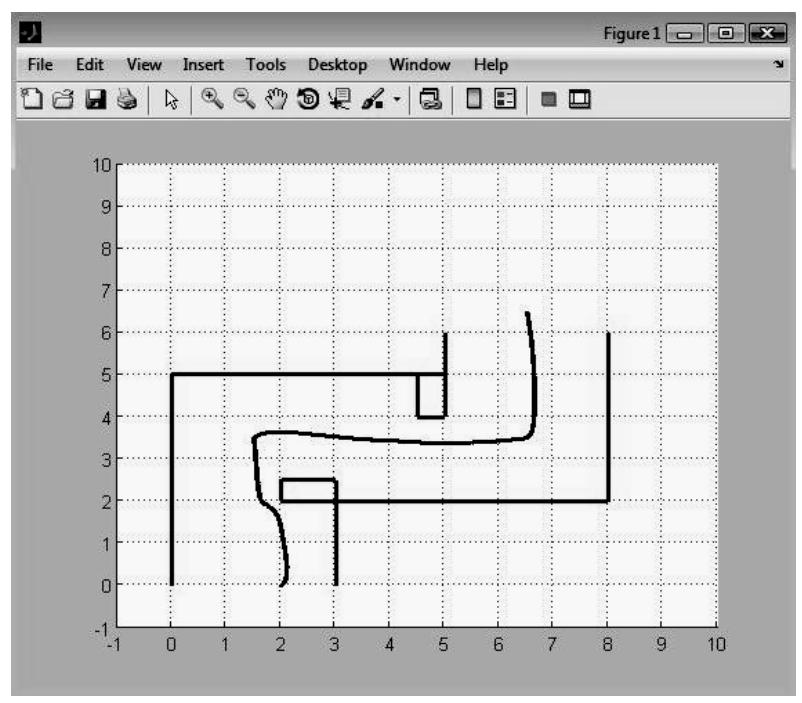

(b)

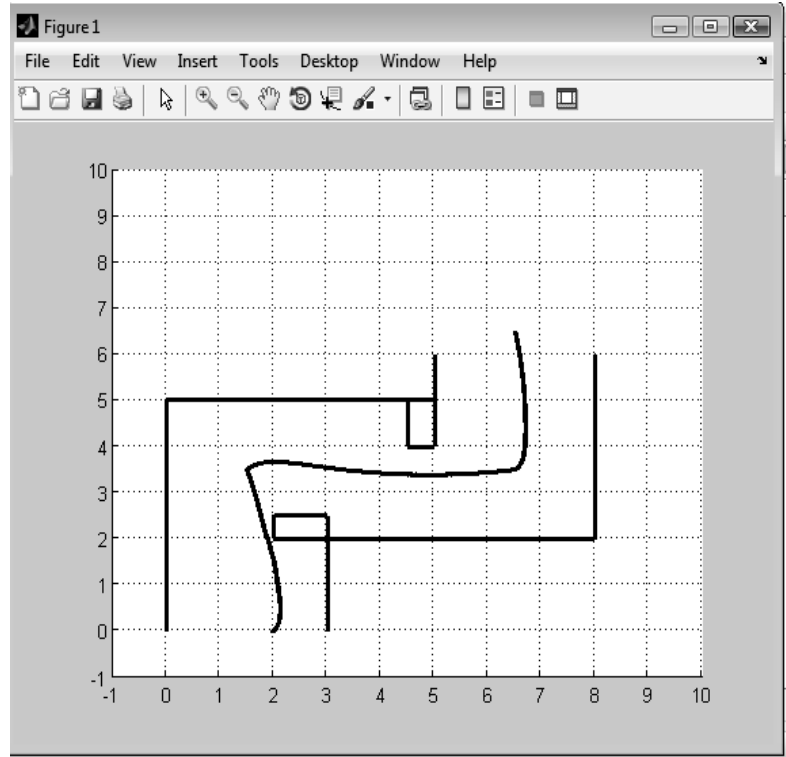

(c)

Fig 4: Robot paths in Case 4 with different systems. (a) using APF without GA in [10], (b) using APF with GA in [11], and (c) the proposed system using AAPF with GA.

\subsection{Robot Motion to reach a Static Goal using AAPF controller with the average values of $\mathrm{Ka}$ and $\mathrm{Kr}$ at the four \\ workspaces}

The average values of $\mathrm{Ka}$ and $\mathrm{Kr}$ at the previous four cases are $18.0252,0.4023$, respectively. These two average values are applied again onto the four cases. The paths of the robot at these four cases with these average values are shown in Fig. 5. The smoothness achieved at each case using the average values of $\mathrm{Ka}$ and $\mathrm{Kr}$ is shown also in Table 2. The comparison between the results of the existing techniques and the proposed scheme is supported by a graphical data in Fig.6.

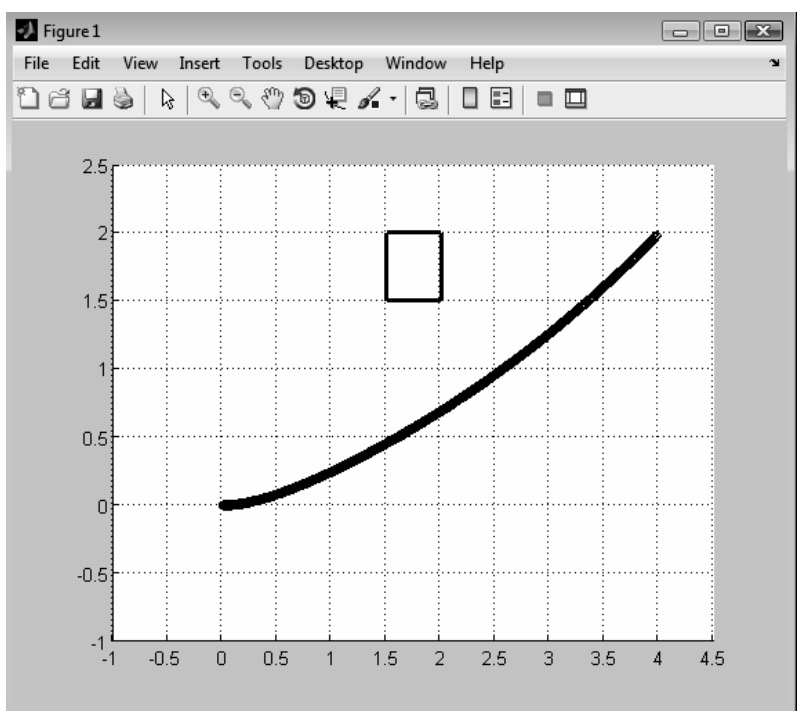

(a) 


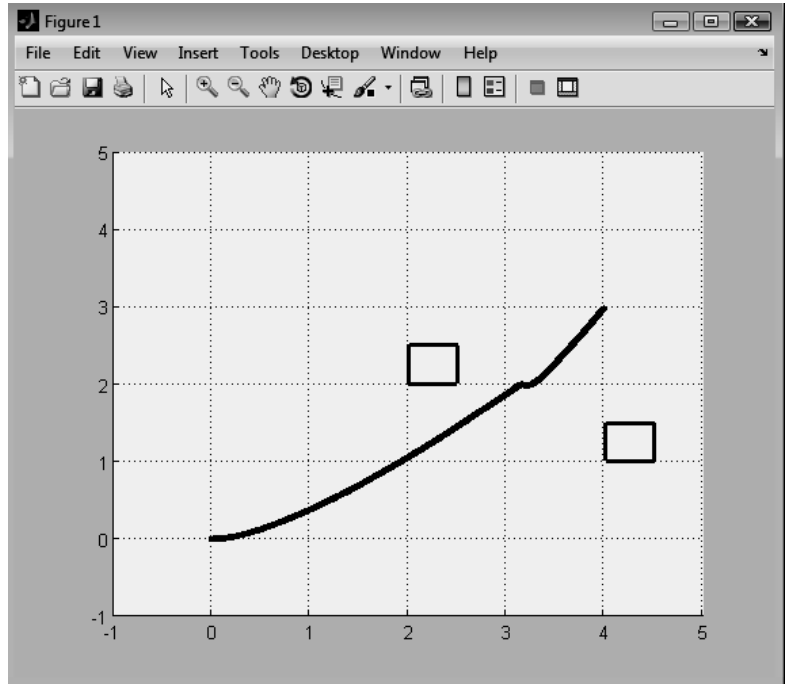

(b)

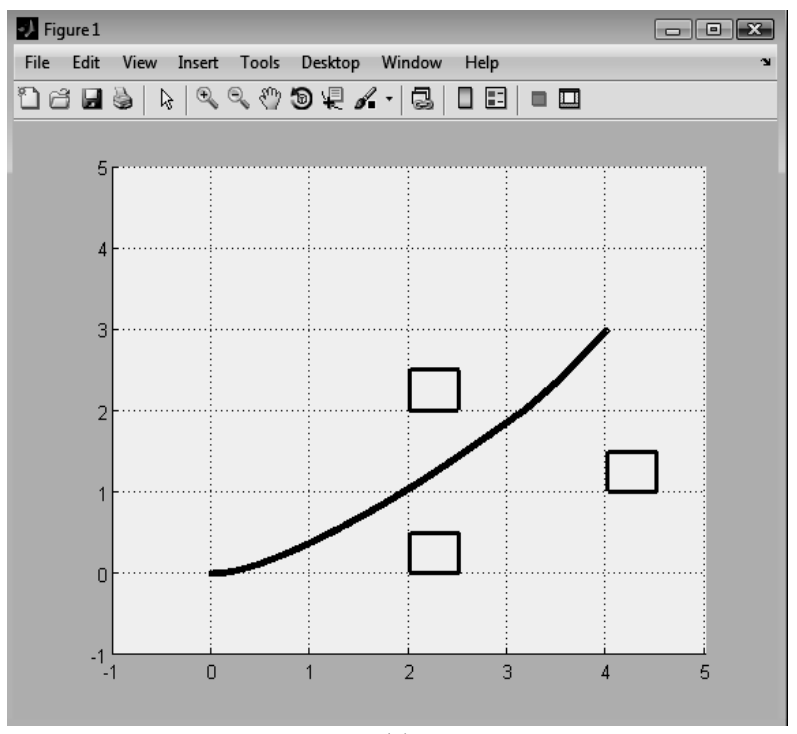

(c)

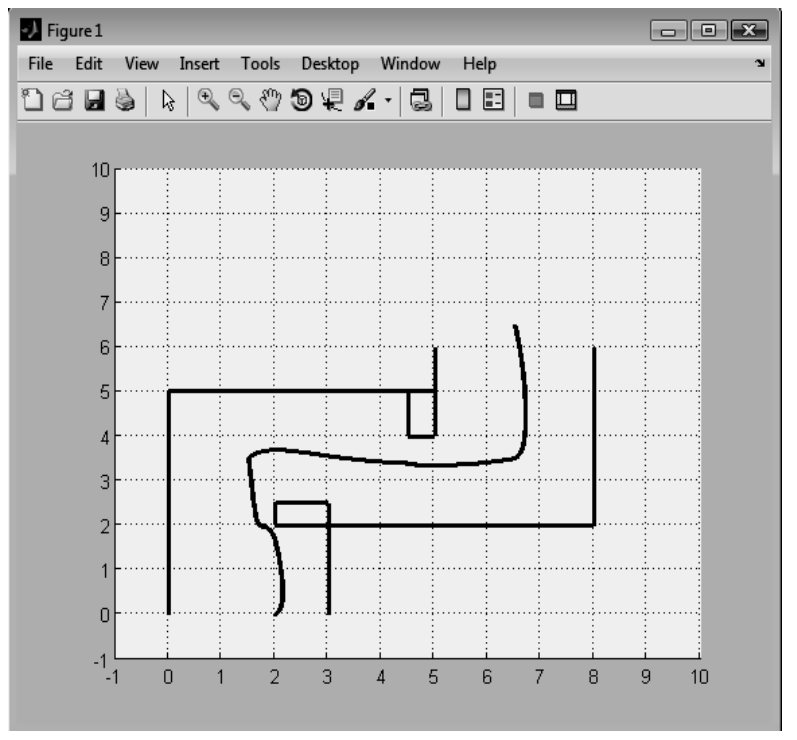

(d)

Fig 5: Robot paths with the average values of $\mathrm{Ka}$ and $\mathrm{Kr}$
Table 2. The comparison of smoothness between the proposed method using AAPF with GA and the previous works

\begin{tabular}{|c|c|c|c|c|}
\hline $\begin{array}{c}\text { Smooth- } \\
\text { ness }\end{array}$ & Case 1 & Case 2 & Case 3 & Case 4 \\
\hline $\begin{array}{c}\text { APF } \\
\text { Without } \\
\text { GA [10] }\end{array}$ & 133.7309 & 303.4949 & 510.1164 & 1420.295 \\
\hline $\begin{array}{c}\text { APF With } \\
\text { GA [11] }\end{array}$ & 0.6268 & 0.9614 & 0.9803 & 54.0509 \\
\hline $\begin{array}{c}\text { AAPF With } \\
\text { GA } \\
\text { [proposed] }\end{array}$ & 0.6503 & 1.0329 & 0.9088 & 17.9618 \\
\hline $\begin{array}{c}\text { AAPF } \\
\text { Using } \\
\text { Average } \\
\text { Factors }\end{array}$ & 0.6876 & 22.224 & 13.9563 & 96.2064 \\
\hline
\end{tabular}

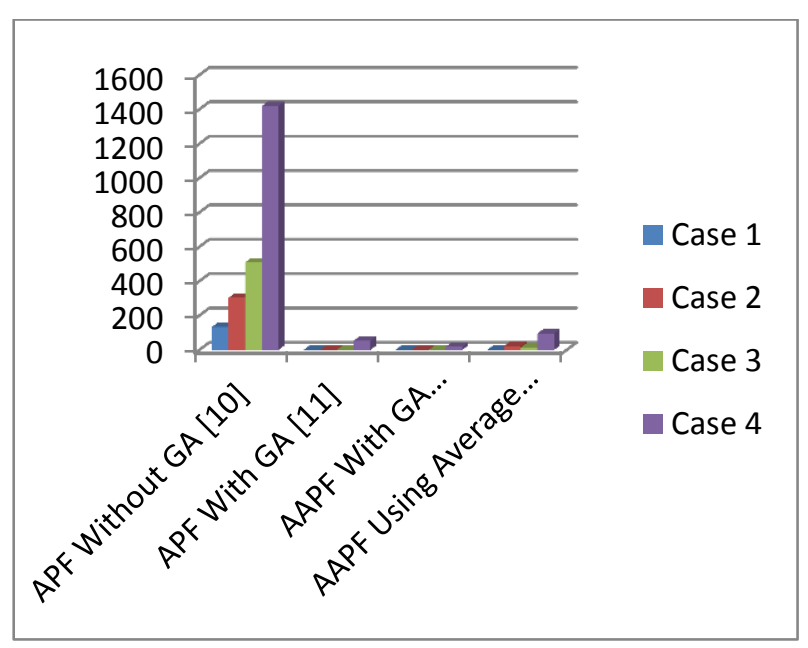

Fig 6: A graphical data illustrates the comparison in table 2.

\section{CONCLUSION}

In this paper, Modification of artificial potential field equation is introduced. Of course this modification gives less computation. Then, the reaction of the robot will achieve higher speed than the robot that uses a traditional artificial potential field controller. GA optimizer is used in different robot environments to select the optimum factors of the forces to reach the goal while avoiding obstacles. The optimized parameters are tested in four different environments. The results show the superiority of the proposed controller generally and inclusively compared to the work in [10]. The average of the computed factors by GA optimizer for the four cases is applied again onto the same cases, while the smoothness in our work remains better than the results in [10]. Graceful optimization regarding the path motion is achieved. The potential fields controlling the robot motion are: attractive one (from the goal), and repulsive one from the obstacles. The real implementation of the proposed work and robots formation can be done as a future work.

\section{REFERENCES}

[1] O. Khatib, "Real-Time Obstacle Avoidance for Manipulators and Mobile Robots", Int. J. Robotics Research, Vol. 5, No. 1, Spring 1986, pp. 90-98. 
[2] J. Borenstein and Y. Koren, "The Vector Field Histogram-Fast Obstacle Avoidance for Mobile Robots", IEEE Trans. on Robotics Automation, Vol. 7, No. 3, June 1991, pp. 278-288.

[3] J. Borenstein and Y. Koren, "Obstacle Avoidance with Ultrasonic Sensors", IEEE Journal of Robotics, Vol. 4, No. 2, 1988, pp. 213-218.

[4] E. Shi, T. Cai, C. He and J. Guo, "Study of the New Method for Improving Artificial Potential Field in Mobile Robot Obstacle Avoidance", Proc. of IEEE International Conference on Automation and Logistics, 2007, pp 282-286.

[5] J. Gong, Y. Duan, Y. Man, and G. Xiong, "VPH+: An Enhanced Vector Polar Histogram Method for Mobile Robot Obstacle Avoidance", Proc. of International Conference on Mechatronics and Automation, 2007, pp. 2784-2788.

[6] R. G. Simmons, "The Curvature-Velocity Method for Local Obstacle Avoidance", Proc. of IEEE International Conference on Robotics and Automation, April 1996, pp.3375-3382.

[7] N. Y. Ko, R. G. Simmons, and K. S. Kim, "A Lane Based Obstacle Avoidance Method for Mobile Robot Navigation", Journal of Mechanical Science and Technology, Vol. 17, No. 11, 2003, pp. 1693-1703.
[8] A. Kelly, "An Intelligent Predictive Control Approach to the High Speed Cross Country Autonomous Navigation Problem", Tech Report CMU-CS-TR-95-33, School of Computer Science, Carnegie Mellon University, 1995.

[9] C. Schlegel, "Fast Local Obstacle Avoidance under Kinematic and Dynamic Constrains for a Mobile Robot", Proc. of IEEE/RSJ International Conference on Intelligent Robots and Systems, 1998, Vol. 1, pp. 594599.

[10] Dong Jin Seo, Nak Yong Ko, and Jung Eun Son, "A Method for Combining Odometry and Distance Sensor Information for Effective Obstacle Avoidance of Autonomous Mobile Robots", International Journal of Control, Automation, and Systems, Vol. 8, No. 3, 2010, pp. 597-603.

[11] Marwa Taher, Hosam Eldin Ibrahim, Shahira Mahmoud, Elsayed Mostafa, "Tracking of a Moving Target by Improved Potential Field Controller in Cluttered Environments", IJCSI International Journal of Computer Science Issues, Vol. 9, Issue 2, No 3, March 2012, pp. $472-480$.

[12] Philip Machler, "Robot Positioning by Supervised and Unsupervised Odometry Correction", PhD thesis, Department of Information, École Poly Technique Federal of Lausanne, 1998. 